\title{
Gendering the Machine: Preferred Virtual Assistant Gender and Realism in Self-service
}

\author{
Jeunese Payne ${ }^{1}$, Andrea Szymkowiak ${ }^{1}$, Paul Robertson ${ }^{1}$, and Graham Johnson ${ }^{2}$ \\ ${ }^{1}$ University of Abertay Dundee, Dundee DD1 1HG, UK \\ 0501726@live.abertay.ac.uk,a.szymkowiak@abertay.ac.uk, \\ p.robertsoneabertay.ac.uk \\ ${ }^{2}$ NCR Discovery Centre, 3 Fulton Road, Dundee DD2 4SW, UK \\ graham. johnsonencr. com
}

\begin{abstract}
A virtual agent is a human-like character that is designed to assist users in interactions with technology and virtual worlds. Research into the preferred visual characteristics of a virtual agent has focused on education-based agents, gaming avatars, and online help assistants. However, findings from these studies are not necessarily generalizable to other technologies, such as self-service checkouts (SSCO). This paper describes data from 578 participants, looking at the gender preferences of Virtual Assistants (VA) in a SSCO context and the impact of VA realism depending on user gender. Due to female participants' preference for female VAs, and an overall preference for threedimensional characters, a realistic, female VA should be used in SSCO. The results are discussed in terms of similarity-attraction theory and social role theory.
\end{abstract}

Keywords: Interface Agent, Virtual Assistant, Gender, Visual Realism, Selfservice, Psychology, Preference

\section{Introduction}

Technology plays a central role in service delivery, providing several channels through which consumers can be reached, e.g., the Internet, mobile technologies and kiosks [1-2]. Many of these channels are self-service - technologies that enable users to direct the service they receive, e.g., cash withdrawals, shopping transactions etc., with minimal direct input from staff [3].

Self-service technology tends to be used less by people who value the social interaction available during face-to-face transactions [4]. This suggests that SSCO would benefit from social presence, i.e., the feeling of interacting with another person such that the perceived role of technology is reduced [5-7]. Reeves and Nass [8] argue that people already treat technologies like social actors - a tendency that could be enhanced in SSCOs by implementing a human-like interface agent in the role of Virtual Assistant (VA). Additionally, a visually present VA can improve performance by reducing the number of people that make mistakes during transactions [9].

Most research into self-service technology has focused on the attitude towards and interaction with websites [4], and thus the impact of virtual characters has focused on

adfa, p. 1, 2011.

(C) Springer-Verlag Berlin Heidelberg 2011 
their role in online applications [6], [10-15]. It is difficult to determine whether the effects of particular services investigated in one study can be generalized to other types of services [2], as users of different technology-based services have different motivations and rely on different types of guidance based on the context and task.

\subsection{Background}

Technology-based interaction with consumers reduces the capacity to meet the need for social interaction during service encounters, having a negative impact on the consumer's intention to use self-service technology [2]. An appropriate visual representation of an employee could reintroduce a positive social aspect. Conversely, a virtual agent that lacks perceived intelligence, competence or trustworthiness, or that appears eerie, unattractive, inappropriate or unhelpful, has the potential for negative outcomes such as frustration or unwillingness to use it [14], [16].

Enabling users to select an agent's appearance should provide a more veridical index of their preferences rather than requiring users to rate an agent based on critical features. This study looks at preferences for such an agent's gender and degree of realism. In this self-service context, we use the term 'VA' - a specific type of interface agent usually applied to e-commerce. An interface agent is, more generally, a computer-generated character designed to interact with users by simulating human appearance and behaviors through artificial intelligence. Dependent on their function, they are referred to as Virtual Customer Service Agent [15], Product Recommendation Agent [6], Embodied Conversational Agent [11], [17], Pedagogical Agent [18], [19-21], [31], or as a human-like or embodied agent/character [12-13], [23-27].

Research on user preferences for agent gender presents contradictory findings and trends, from a preference for same-sex virtual agents, to a preference for opposite sex virtual agents, and no gender preference at all [6], [17], [22], [28]. These differences may be due to user characteristics, but also context [28-29]. Lee [23] for example, found that participants were more open to suggestions made by a virtual character whose implied gender matched the gendered topic. In a gender-neutral task (sorting photographs) Cowell and Stanney [17] reported a trend in females towards a preference for a virtual character of the opposite sex. Conversely, Kim and Wei [22] found a preference for gender-matched virtual agents, even though the context - a computerbased algebra learning environment - may have been perceived as requiring male expertise. Baylor and Plant [28], however, revealed preferences for gender-matching in females dependent on the topic of the learning task; when the purpose of the agent was to aid users in a male-dominated topic (engineering), male agents were deemed more appropriate and similarity became less important than perceived expertise.

In online retail environments, Qiu and Benbasat [6] suggested that gender is less important to users than other demographics, finding no overt VA gender preference in an online shopping context, but rather a preference for ethnicity-matching, especially in females. McBreen et al. [11] also found little evidence of gender preference. VA gender preference may also be affected by other appearance-based differences. McBreen \& Jack [12], for example, reported that VA gender preference in an e-retail 
environment might be affected by its realism: participants preferred a realistic male over a realistic female agent, and a cartoonized female over a cartoonized male agent.

Virtual Assistant Characteristics. To systematically assess preferences for particular VA characteristics in SSCO, we invited participants to choose among VAs in a public technology exhibit. This helps to determine whether people prefer the idea of interacting with a male or female VA, and the extent to which he/she should resemble actual checkout staff, as opposed to idealized versions of the user, as tends to be the case for role/social models in learning environments [21], [28-30] and selfrepresentations in shared virtual environments ('avatars') [29], [31-32].

Similarity Attraction Theory vs. Social Role Theory. There are two key theories relevant to VA characteristics. The first is similarity-attraction theory, which suggests that people are attracted to similar others [6], [20], [26-27], who tend to be perceived as more credible, resulting in increased liking [6], and in technology, reduced discomfort with the interface [33]. Similarity can be investigated on several levels, including global features such as culture, ethnicity and gender, local features such as hair color and face shape, social and personality factors such as levels of politeness or extraversion, and behavioral similarity [31]. We focus on gender and realism, since these can be directly compared to user characteristics. As it is necessary for human-like characters to be dressed, clothing was varied, but examined in an exploratory manner.

A VA created to give impressions of warmth and expertise may be deemed more appropriate in SSCOs. This is in line with the second theory of relevance, social role theory, which posits that social group roles are used to infer stereotypes about them, reflected in status and power differences [34], [35-36]. For instance, the disproportionate amount of domestic roles that women play relative to men results in the expectation that women should possess communal traits and behaviors such as friendliness. Deviations from gender norms tend to be met with surprise or moral disapproval [35]. In the example of SSCO, a warm (female) and competent (realistic) VA may be preferred, depending on stereotypical expectations of staff in such service encounters.

Gender. Similarity-attraction theory predicts preferences for gender-matching. This may be especially the case for females [6], [32] who have been found to prefer interacting with other females [37], not necessarily because they like females more than males, but because males, as a dominant group, pose more threat [38]. As a result of women's greater inter-dependence (relative to male independence), they tend to use similarity as the basis for social bonds [34]. Based on similarity-attraction theory, two hypotheses are derived with regards to VA and participant gender:

- Hypothesis 1a: Participants will more often choose VAs of the same implied gender to themselves.

- Hypothesis 1b: Female participants will show a greater preference for gendermatching than male participants. 
Social role theory predicts a preference for VAs that represent typical checkout staff, given their role. Females are stereotyped as being caring/helpful [37], and thus, female VAs may be seen as more appropriate in SSCOs than male VAs, i.e., VAs by evoking stereotypes of helpfulness/approachability as deemed suitable for the role:

- Hypothesis 2: Participants will more often choose female VAs over male VAs.

Realism. Hypotheses are harder to derive in relation to realism because it is not possible to compare preferences of a "less realistic person" with preferences of a "more realistic person". It is also difficult to assess in relation to the two theories because a preference for the VA to be most like oneself or most like an actual employee would both result in a preference for high realism.

Unlike gaming worlds and computer-based learning environments, a VA in a SSCO may be more valued as task-oriented, as opposed to socially-oriented. This suggests a preference for realistic, three-dimensional VAs that are similar to users in terms of human-likeness and thus imply a similar level of intelligence and credibility. The reasoning is that while people are drawn to similar others, they do not need others to $b e$ or to represent themselves. Similarly, social role theory predicts a preference for realistic VAs because they are most like actual employees in human-likeness, implying more competence in their role. Based on both theories, we suggest:

- Hypothesis 3: Participants will more often choose more realistic VAs over cartoonized VAs.

Social role theory suggests that this may be especially true for males who value utilitarian aspects of human-computer interaction [6], making the potency of the VA role more important to this demographic of user. Baylor and Kim [39] found that males, but not females, learn more with realistic agents than cartoon agents. Similarly, Haake \& Gulz [18] found that males, who preferred more detailed, three-dimensional agents over cartoon agents, also preferred more strictly task-oriented agents. Thus:

- Hypothesis 4: Male participants will show a greater preference for more realistic VAs than female participants.

\section{$2 \quad$ Method}

Setting. The impact of VA gender and realism was tested by asking participants to choose between VAs that differed on these aspects. Data were obtained from a SSCO simulation (Fig. 1) displayed at the Dundee Science Centre Exhibition titled "Robot: The Fantasy \& the Reality" which was open to the general public. Amongst other attractions, such as robot models used in popular media and robots in science, visitors could interact with the touch-screen on a SSCO typically found in supermarkets. The exhibit stated that participant responses would be used for research and that individual 
data would be anonymous. After selecting a VA, users could then choose to engage in a produce-finding task. The preference data are the focus of this paper.

This method allowed us to collect data from a large participant sample, reducing the margin of sampling error. It may be argued that the sample of visitors to a science center are not representative of the general population. However, a wide variety of individuals from the UK visited the Centre; the exhibition consisted of a variety of exhibits, from robots, to games and showcases of industry-specific technology. Those using the SSCO exhibit are likely to be self-selected - just as users of actual SSCOs are. It is also possible that some used the SSCO more than once or gave incorrect demographic information. The large sample size limits the effects of noise in the data. Also, there was no experimenter present while people interacted with the SSCO, reducing social response bias.
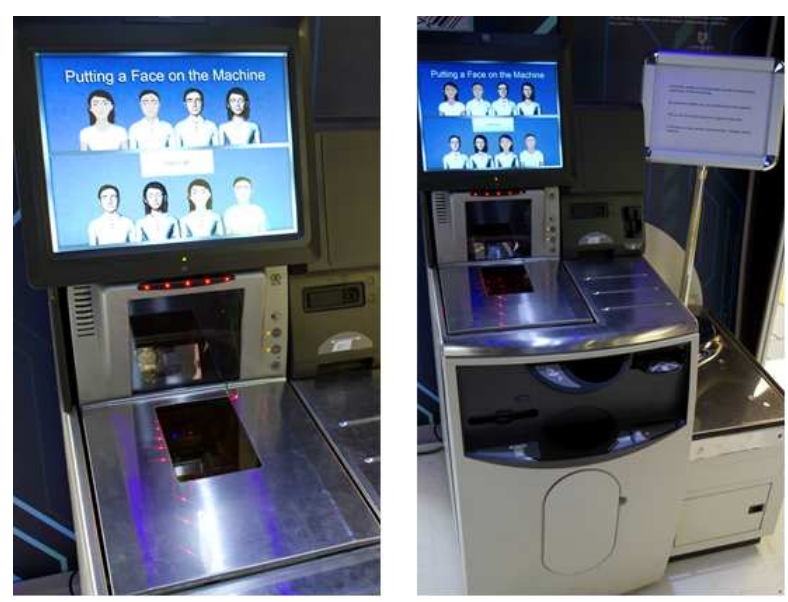

Fig. 1. The SSCO attraction in place at the Dundee Science Centre

Participants. Visitors attending the Dundee Science Centre in the first two weeks of June 2012 were the participant target group. Interested visitors could take part; none were specifically asked to do so. A total of 578 adults (220 male, 358 female) between the ages of 16 and 84 chose a VA (mean age 35.56 years).

Stimuli. Eight static VAs were presented in random order, varying in terms of gender, realism, and formality of dress (Fig. 2). Given the high number of permutations, randomization ensured that systematic effects of VA order were minimal. Formality of dress was an exploratory variable, yielding no significant impact on preference.

We collated the frequency of preferences for male versus female VAs, and the three-dimensional versus cartoonized VAs, and grouped these according to participant gender. Examples of the differences between VAs based on gender and realism can be seen in Fig. 2. 
Procedure. After reading the start-up page providing information on the study, participants pressed the 'Begin' button, and eight VAs were displayed on the screen. Participants were asked to choose their preferred VA by touching its image (Fig. 2). Participants were then asked to give demographic information, followed by the produce finding task. The frequency of VA choices was logged as the Dependent Variable.

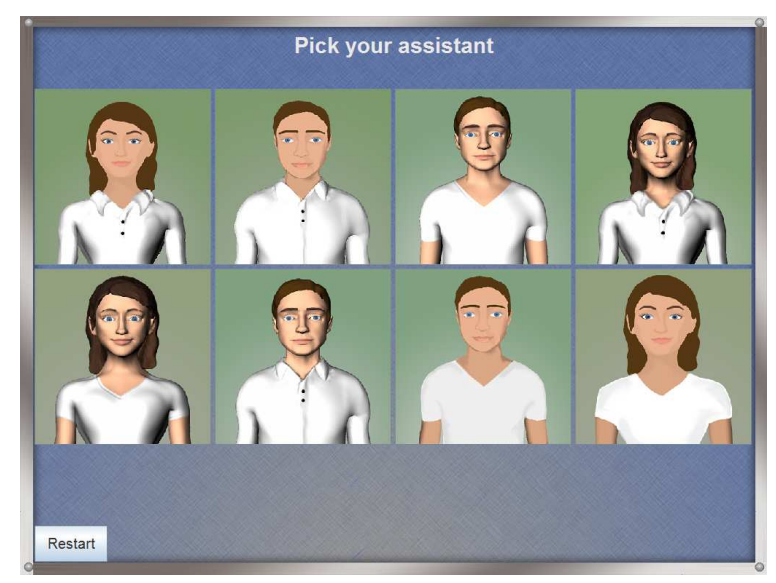

Fig. 2. Eight VAs differing in gender, realism, and dress (exploratory)

Results - VA Gender. Addressing Hypotheses 1a, 1b, and 2, a 2x2 Chi-Square test revealed a relationship between the VA's implied gender and participant gender $[\chi 2(1, \mathrm{~N}=578)=78.364, \mathrm{p}<0.001]$ with a moderate association $[\Phi=0.368]$. The majority $(\approx 83 \%)$ of females chose a female VA, compared to nearly half $(\approx 48 \%)$ of males (Table 1). Female VAs were preferred over male VAs with almost $70 \%$ of all participants choosing a female VA.

Table 1. The Count and Percentages of Male and Female Preferences for VA Gender

\begin{tabular}{|l|c|c|c|}
\hline & $\begin{array}{c}\text { Participant } \\
\text { Female }\end{array}$ & Participant Male & Total \\
\hline VA Female & 296 & 105 & 401 \\
& $(82.7 \%)$ & $(47.7 \%)$ & $(69.4 \%)$ \\
\hline VA Male & 62 & 115 & 177 \\
& $(17.3 \%)$ & $(52.3 \%)$ & $(30.6 \%)$ \\
\hline Total & 358 & 220 & 578 \\
\hline
\end{tabular}

Results - VA Realism. Addressing Hypotheses 3 and 4, a 2x2 Chi-Square test revealed a relationship between VA realism and participant gender $[\chi 2(1, \mathrm{~N}=578)=$ 
8.093, $\mathrm{p}=0.004]$ with a weak association: $\Phi=0.118$. The majority $(\approx 63 \%)$ of females but more males $(\approx 75 \%$ ) chose three-dimensional, realistic options (Table 2 ).

Table 2. The Count and Percentages of Male and Female Preferences for VA Realism

\begin{tabular}{|l|c|c|c|}
\hline & $\begin{array}{c}\text { Participant } \\
\text { Female }\end{array}$ & Participant Male & Total \\
\hline Cartoonized VA & $\begin{array}{c}132 \\
(36.9 \%)\end{array}$ & $\begin{array}{c}56 \\
(25.5 \%)\end{array}$ & $\begin{array}{c}188 \\
(32.5)\end{array}$ \\
\hline 3-dimensional VA & 226 & 164 & 390 \\
& $(63.1)$ & $(74.5 \%)$ & $(67.5 \%)$ \\
\hline Total & 358 & 220 & 578 \\
\hline
\end{tabular}

\section{Discussion}

Similarity-attraction theory predicts that participants would choose to gender-match. This was found for the majority of females, but not males, who chose male VAs almost as often as they chose female VAs, supporting Hypotheses 1a partially and $1 \mathrm{~b}$ fully. Overall preference was for female VAs, but because this relies primarily on female participant data, the findings only partially support Hypothesis 2. The male gender-preference data were in line with neither similarity-attraction theory, nor social role theory. The findings also support Hypothesis 3, as most participants chose three-dimensional VAs, reinforcing the idea that VAs in a SSCO context serve a taskbased function, with slightly more men than women preferring this level of realism (support for Hypothesis 4), lending support for social role theory.

While neither theory was fully supported, they provide insights and reasonable interpretations of the findings. Similarity-attraction theory suggests that identification with a social group enhances co-operative behavior, commitment, knowledge contribution, participation, and organizational citizenship behavior [7]. Females, having a stronger collective bond with other females than males have with other males [37], might have chosen to gender-match because it increases comfort during the interaction. There may also be positive performance-based outcomes for female users interacting with a same sex VA, as evidence suggests that women become more taskoriented when interacting in same-sex groups than in mixed-sex groups [33-34]. In contrast, males often like interacting with the opposite sex, due to positive attitudes towards maternal attributes [37]. Despite this, our results show no male preference for a female VA. The findings reflect a less pronounced within-sex bias in men, consistent with other research in human-human interaction [37].

The findings are also consistent with research suggesting that males are more concerned with utilitarian aspects of human-computer interaction [6], [39], since a higher percentage of males than females chose a realistic agent. Nevertheless, the majority of both males and females preferred three-dimensional, realistic VAs. Thus, both sexes seemed more concerned with utilitarian aspects of the SSCO simulation, and less concerned with hedonic aspects. While Haake and Gulz [18] found no preference for 
realism in males, they did find that males who preferred more detailed, threedimensional agents over cartoon agents also preferred more strictly task-oriented agents - highlighting the link between realism and utilitarian goals.

More females preferred realistic VAs than cartoonized Vas, suggesting that human-likeness (similarity-attraction) and/or assistance-based role (social role) of VAs were more important to users over other factors, such as self-representation or enjoyment. The two possible explanations based on the two theories are not necessarily in opposition. First, in line with similarity-attraction theory, participants of both sexes may have been driven by the task-oriented nature of SSCO, resulting in a preference for VAs that were most like themselves in terms of realism. Second, in line with social role theory, participants may have been more concerned with the VA's role over its attractiveness, thus, choosing VAs that were closer visual representations of actual supermarket employees, i.e., three-dimensional. In SSCO, this involves displaying 'appropriate' checkout assistant characteristics such as warmth and approachability traits which are more readily perceived in human-like females [36].

There are some limitations associated with this study. First, perceptions of the VA were not measured. These perceptions, and other possible reasons for choosing VAs, could be tackled in future studies, perhaps with more qualitative research. Second, there may have been other factors that made three-dimensional VAs more attractive, such as familiarity with realistic characters in computer games or the association between two-dimensional cartoons with children's TV programs. Realistic VAs could also have been more attractive because they were more (but not too) human-like, thus avoiding the "Uncanny Valley", which proposes that more human-like characters are preferred to less human-like characters, until they become so human-like that they are eerie [14], [16]. Finally, the context is less ecologically valid than an actual supermarket setting, but arguably more ecologically valid than a lab-based study.

Conclusions. Females chose to gender-match and to interact with a more realistic VA. Males exhibited little preference for either gender, and a greater preference than females for realistic VAs. Thus, where it is not feasible to gender-match in SSCO, the recommendation is to implement a realistic female VA.

Our findings raise the question of whether VA choice would be a viable option to engage consumers in SSCO, as this would go beyond mere acceptance of an existing agent, thus potentially alleviating the perpetuation of stereotypical assumptions.

Acknowledgments. We thank the Dundee Science Centre for exhibiting our SSCO. Thanks go to all participants for taking part, Rosie Henderson (Abertay University) for the creation of the VAs and Stuart Birse (NCR) for providing the pictures of the SSCO set-up. Finally, we thank NCR for funding this research.

\section{References}

1. Rust, R.T., Espinoza, F.: How Technology Advances Influence Business Research and Marketing Strategy. J. Bus. Res. 59, 1072-1078 (2006) 
2. Simon, F., Usunier, J-C.: Cognitive, Demographic, and Situational Determinants of Service Customer Preferences for Personnel-in-contact over Self-service Technology. International J. Res. in Market. 24, 163-173 (2007)

3. Meuter, M.L., Bitner, M.J., Ostrom, A.L., Brown, S.W.: Choosing among Alternative Service Delivery Modes: An Investigation of Customer Trial of Self-service Technologies. J. Market. 69, 61-83 (2005)

4. Lee, H-J., Fairhurst, A., Cho, H.J.: Gender Differences in Consumer Evaluations of Service Quality: Self-Service Kiosks in Retail. The Serv. Industries J. 33, 248-265 (2013)

5. Abeele, M.V., Roe, K., Pandelaere, M.: Construct Validation of the Concepts Social Presence, Emotional Presence and Connectedness and an Application of Zajonc's Social Facilitation Theory to Social Presence Research. In: Proceedings of the 10th Annual International Workshop on Presence, pp. 215-224 (2007).

6. Qiu, L., Benbasat, I.: A study of Demographic Embodiment of Product Recommendation Agents in Electronic Commerce. Int. J. Hum.-Comp. Stud. 68, 669-688 (2010)

7. Shen, K. N., Yu, A. Y., \& Khalifa, M.: Knowledge Contribution in Virtual Communities: Accounting for Multiple Dimensions of Social Presence through Social Identity. Beh. Inf. Tech. 29, 337-348 (2009)

8. Reeves, B., Nass, C.: The Media Equation: How People Treat Computers, Television, and New Media Like Real People and Places. CSLI Publications, Cambridge (1996)

9. Payne, J.A., Johnson, G.I., Szymkowiak, A.:The Behavioural Impact of a Visually Represented Virtual Assistant in a Self-Service Checkout Context. In: Proceedings of the 25th BCS Conference on Human-Computer Interaction (pp. 58-63). British Computer Society (2011)

10. Heckman, C.E., Wobbrock, J.O.: Put Your Best Face Forward: Anthropomorphic Agents, E-Commerce Consumers, and the Law. In: Proceedings of the ACM Conference on Autonomous Agents, pp. 435-442 (2000)

11. McBreen, H., Anderson, J., Jack, M.: Evaluating 3D Embodied Conversational Agents in Contrasting VRML Retail Applications. Proceedings of International Conference of Autonomous Agents Workshop on Multimodal Communication and Context in Embodied Agents, 83-87 (2001)

12. McBreen, H.M., Jack, M.A.: Evaluating Humanoid Synthetic Agents in E-Retail Applications. IEEE Transactions on Systems, Man, and Cybernetics 31, 394-405 (2001)

13. Brave, S., Nass, C., Hutchinson, K.: Computers That Care: Investigating the Effects of Orientation of Emotion Exhibited by an Embodied Computer Agent. Int. J.. Hum.-Comp. Stud. 62, 161-178 (2005)

14. Groom, V., Nass, C., Chen, T., Nielsen, A., Scarborough, J.K., Robles, E.: Evaluating the Effects of Behavioral Realism in Embodied Agents. Int. J. Hum.-Comp. Stud. 67, 842-849 (2009)

15. Verhagen, T., van Nes, J., Feldberg, F., van Dolen, W.: Virtual customer service agents: Using social presence and personalization to shape online service encounters. Res. Memorand. 10 (2011)

16. MacDorman, K.F., Green, R.D., Ho, C-C., Koch, C.T.: Too Real for Comfort? Uncanny Responses to Computer Generated Faces. Comp. Hum. Beh. 25, 695-710 (2009)

17. Cowell, A.J., Stanney, K.M.: Manipulation of Non-verbal Interaction Style and Demographic Embodiment to Increase Anthropomorphic Computer Character Credibility. Int. J. Hum.-Comp. Stud. 6, 281-306 (2005)

18. Haake, M., Gulz, A.: A Look at the Roles of Looks \& Roles in Embodied Pedagogical Agents - A User Preference Perspective. Int. J. Art. Int. Ed. 19, 39-71 (2009) 
19. Sahimi, A.M., Zain, F.M., Kamar, N.A.N., Samar, N., Rahman, Z.A., Majid, O., Atan, H., Fook, F.S.: The Pedagogical Agent in Online Learning: Effects of the Degree of Realism on Achievement in Terms of Gender. Contemp. Educ. Technol. 1, 175-185 (2010)

20. Veletsianos, G.: Contextually Relevant Pedagogical Agents: Visual Appearance, Stereotypes, and First Impressions and Their Impact on Learning. Comp. \& Educ. 55, 576-585 (2010)

21. Baylor, A.L.: The Design of Motivational Agents and Avatars. Educ. Tech. Res. \& Develop. 59, 291-300 (2011)

22. Kim, Y., Wei, Q.: The Impact of Learner Attributes and Learner Choice in an Agent-based Environment. Comp. Educ. 56, 505-514 (2011)

23. Lee, E-J.: The Effects of "Gender" of the Computer on Informational Social Influence: The Moderating Role of Task Type. Int. J. Hum.-Comp. Stud. 58, 347-362 (2003)

24. Berry, D.C., Butler, L.T., de Rosis, F.: Evaluating a Realistic Agent in an Advice-giving Task. Int. J. Hum.-Comp. Stud. 63, 304-327 (2005)

25. Gong, L.: Is Happy Better than Sad even if They Are Both Non-Adaptive? Effects of Emotional Expressions of Talking-Head Interface Agents. Int.J. Hum.-Comp. Stud. 65, 183191 (2007)

26. Pratt, J.A. Hauser, K., Ugray, Z., Patterson, O.: Looking at Human-computer Interface Design: Effects of Ethnicity in Computer Agents. Inter. Comp. 19, 512-523 (2007)

27. Van Vugt, H.V., Konijn, E.A., Hoorn, J.F., Keur, I., Eliens, A.: Realism is Not All! User Engagement with Task-Related Interface Characters. Inter. Comp. 19, 267-280 (2007)

28. Baylor, A.L., Plant, E.A.: Pedagogical Agents as Social Models for Engineering: The Influence of Agent Appearance on Female Choice. Art. Intell. Ed. 125, 65-72 (2005)

29. Baylor, A.L.: Promoting Motivation with Virtual Agents and Avatars: Role of Visual Presence and Appearance. Phil.1 Trans. Royal Soc. B, 364(1535), 3559-3565 (2009)

30. Rosenberg-Kima, R.B., Baylor, A.L., Plant, E.A., Doerr, C.E.: Interface Agents as Social Models for Female Students: The Effects of Agent Visual Presence and Appearance on Female Students' Attitudes and Beliefs. Comp. Hum. Beh. 24, 2741-2756 (2008)

31. Bailenson, J.N., Yee, N.: Digital Chameleons: Automatic Assimilation of Nonverbal Gestures in Immersive Virtual Environments. Psych. Sc. 16, 814-819 (2005)

32. Ducheneaut, N., Wen, M-H., Yee, N., Wadley, G.: Body and Mind: A Study of Avatar Personalization in Three Virtual Worlds. Proceedings of CHI, 2009.

33. Li, I., Forlizzi, J., Dey, A., Kiesler, S.: When the Interface is the User's Face: Ideas for Research And Applications. CHI Workshop in HCI and the Face. 2006

34. Cross, S.E., Madson, L.: Models of the Self: Self-construals and Gender. Psych. Bull. 122, 5-37 (1997).

35. Eagly, A.H., Wood, W., Diekman, A.B.: Social Role Theory of Sex Difference and Similarities: A Current Appraisal. In: Eckes, T., Trautner, H.M. (eds.) Developmental Social Psychology of Gender. Lawrence Erlbaum Associates, Inc., New Jersey (2000)

36. Cuddy, A.J.C., Fiske, S.T., Glick, P.: The BIAS map: Behaviors from Intergroup Affect and Stereotypes. J. Pers. Soc. Psych. 92, 631-648 (2007)

37. Rudman, L.A., Goodwin, S.A.: Gender Differences in Automatic In-Group Bias: Why Do Women Like Women More than Men Like Men? J. Pers. Soc. Psych. 87, 494-509 (2004)

38. Vigil, J.M.: A socio-relational Framework of Sex Differences in the Expression of Emotion. Beh. Brain Sc. 32, 375-390 (2009)

39. Baylor, A.L., Kim, Y.: Pedagogical Agent Design: The Impact of Realism, Gender, Ethnicity, and Instructional Role. Proceedings of the International Conference in Intelligent Tutoring Systems (ITS), 2004, 592-603 (2004) 